\title{
COMPONENTWISE LINEAR IDEALS
}

\author{
JÜRGEN HERZOG AND TAKAYUKI HIBI
}

\begin{abstract}
A componentwise linear ideal is a graded ideal $I$ of a polynomial ring such that, for each degree $q$, the ideal generated by all homogeneous polynomials of degree $q$ belonging to $I$ has a linear resolution. Examples of componentwise linear ideals include stable monomial ideals and Gotzmann ideals. The graded Betti numbers of a componentwise linear ideal can be determined by the graded Betti numbers of its components. Combinatorics on squarefree componentwise linear ideals will be especially studied. It turns out that the Stanley-Reisner ideal $I_{\Delta}$ arising from a simplicial complex $\Delta$ is componentwise linear if and only if the Alexander dual of $\Delta$ is sequentially Cohen-Macaulay. This result generalizes the theorem by Eagon and Reiner which says that the StanleyReisner ideal of a simplicial complex has a linear resolution if and only if its Alexander dual is Cohen-Macaulay.
\end{abstract}

\section{Introduction}

Let $A=K\left[x_{1}, x_{2}, \ldots, x_{n}\right]$ denote the polynomial ring in $n$ variables over a field $K$ with each $\operatorname{deg} x_{i}=1$ and $I$ a graded ideal of $A$. The topic of the present paper is computation of graded Betti numbers $\beta_{i, j}=\beta_{i, j}(I)$ which appear in a graded minimal free resolution

$$
0 \longrightarrow \bigoplus_{j \in \mathbf{Z}} A(-j)^{\beta_{h, j}} \longrightarrow \cdots \longrightarrow \bigoplus_{j \in \mathbf{Z}} A(-j)^{\beta_{0, j}} \longrightarrow I \longrightarrow 0
$$

of $I$ over $A$. Let $\beta_{i}=\beta_{i}(I):=\sum_{j \in \mathbf{Z}} \beta_{i, j}$ and call $\beta_{i}$ the $i$-th graded Betti number of $I$. Some fundamental works have been achieved, from view points of both commutative algebra and combinatorics, for ideals generated by monomials. Eliahou and Kervaire [11] constructed an explicit minimal free resolution and computed Betti numbers of stable monomial ideals (see also [1] and [8]). Their computational result is indispensable for Bigatti [5] and Hullet [17], where upper bounds of Betti numbers of graded ideals with a given Hilbert function are discussed. Moreover, the squarefree analogues of [11], [5] and [17] are studied in [2] and [3]. On the other hand, in [21] and

Received November 25, 1996.

Revised February 23, 1998. 
[22], Betti numbers of squarefree monomial ideals associated with boundary complexes of cyclic polytopes and stacked polytopes are computed.

More attention has been paid to graded ideals with linear resolutions. We say that $I$ has a linear resolution if there exists an integer $m \geq 1$ such that $\beta_{i, i+j}=0$ for all $i$ and $j$ with $j \neq m$. Thus, in particular, if $I$ has a linear resolution, then $I$ is generated by homogeneous polynomials of the same degree. When $I=I_{\Delta}$ is the squarefree monomial ideal (e.g., [6], [15] and [19]) arising from a simplicial complex $\Delta$ on the vertex set $[n]=\{1,2, \ldots, n\}$, we know an effective formula (Hochster [16]) to compute Betti numbers of $I$. By virtue of the formula together with Alexander duality theorem, recently, Eagon and Reiner [9] proved the result that $I_{\Delta}$ has a linear resolution if and only if the dual complex $\Delta^{*}$ is Cohen-Macaulay ([9, Theorem 3]). (See also [7] and [23] for some applications of Alexander duality theorem.) Moreover, when $I_{\Delta}$ has a linear resolution, an explicit formula to compute the Betti numbers of $I_{\Delta}$ in terms of the $h$-vector of $\Delta^{*}$ is obtained ([9, Theorem 4]).

In this paper we introduce the notion of componentwise linear ideals. A graded ideal $I \subset A$ is called componentwise linear if, for each degree $q$, the ideal generated by all homogeneous polynomials of degree $q$ belonging to $I$ has a linear resolution. For example, every stable monomial ideal is componentwise linear. We show that graded Betti numbers of a componentwise linear ideal can be determined by the graded Betti numbers of its component (Proposition 1.3). This computational fact enables us to obtain a criterion for a componentwise linear ideal to be a Gotzmann ideal in terms of its graded Betti numbers (Corollary 1.4). We are especially interested in combinatorics on componentwise linear ideals arising from simplicial complexes. Some results on squarefree monomial ideals with linear resolutions obtained by Eagon and Reiner [9] can be generalized to componentwise linear ideals generated by squarefree monomials (Theorem 2.1).

We also apply [9, Theorem 3] to squarefree Gotzmann ideals and conclude this paper with the following combinatorial result: If $\Delta$ is a "pure" simplicial complex of dimension $d-1$ with $f$-vector $\left(f_{0}, f_{1}, \cdots, f_{d-1}\right)$ and if $\partial_{d-1}\left(f_{d-1}\right)=f_{d-2}$, then $\Delta$ is Cohen-Macaulay (cf. Theorem 2.3).

\section{$\S 1$. Algebraic properties of componentwise linear ideals}

Let $A=K\left[x_{1}, x_{2}, \ldots, x_{n}\right]$ denote the polynomial ring in $n$ variables over a field $K$ with each $\operatorname{deg} x_{i}=1$ and $\mathfrak{m}=\left(x_{1}, x_{2}, \ldots, x_{n}\right)$ the graded maximal ideal of $A$. If $I$ is a graded ideal of $A$, then we write $I_{\langle j\rangle}$ for 
the ideal generated by all homogeneous polynomials of degree $j$ belonging to $I$. Moreover, we write $I_{\leq k}$ for the ideal generated by all homogeneous polynomials of $I$ whose degree is less than or equal to $k$.

We say that a graded ideal $I \subset A$ is componentwise linear if $I_{\langle j\rangle}$ has a linear resolution for all $j$.

EXAMPLES 1.1. (a) In the theory of monomial ideal, there is the following hierarchy of ideals: lexsegment monomial ideals $\Rightarrow$ strongly stable monomial ideals $\Rightarrow$ stable monomial ideals. We refer the reader to [5], [11] and [17] for the definitions and the detailed information about these monomial ideals. See also [2]. The reason why lexsegment ideals are important is that, given a graded ideal $I \subset A$, there exists a unique lexsegment ideal $I^{l e x}$ such that $I$ and $I^{\text {lex }}$ have the same Hilbert function. If $I \subset A$ is a stable (resp. strongly stable, lexsegment) ideal, then each $I_{\langle j\rangle}$ is also stable (resp. strongly stable, lexsegment). It follows from [11] that a stable ideal generated by monomials of the same degree has a linear resolution. Hence, a stable monomial ideal is componentwise linear.

(b) A graded ideal $I$ generated in degree $q$ is called a Gotzmann ideal if the number of generators of $\mathfrak{m} I$ is the smallest possible, namely equal to the number of generators of $\mathfrak{m} I^{\text {lex }}$. By Gotzmann's persistence theorem [12] (also see [13]), a graded ideal $I$ generated in degree $q$ is a Gotzmann ideal if and only if $I$ and $\left(I^{l e x}\right)_{\langle q\rangle}$ have the same Hilbert function. Thus, in particular, a Gotzmann ideal has a linear resolution with the same Betti numbers as the corresponding lexsegment ideal. In fact, if $I$ is a Gotzmann ideal generated in degree $q$, then $I^{l e x}$ is also generated in degree $q$. It follows from the Eliahou-Kervaire resolution [11] (see also [1]) yields that $I^{\text {lex }}$ has a linear resolution. By the theorem of Bigatti [5], Hullet [17] as well as Pardue [18], we have $\beta_{i, i+j}(I) \leq \beta_{i, i+j}\left(I^{\text {lex }}\right)$. Thus, we conclude that $I$ has a linear resolution, too. Furthermore, since $I$ and $I^{\text {lex }}$ have the same Hilbert function and both their resolutions are linear, their Betti numbers must coincide.

More general, we call a graded ideal $I \subset A$ a Gotzmann ideal if all component ideals $I_{\langle j\rangle}$ are Gotzmann ideals. Thus, Gotzmann ideals in this new sense are componentwise linear.

The following Lemma 1.2 shows that the part $I_{\leq k}$ of the ideal $I$ determines already a certain range of its graded Betti numbers. 
Lemma 1.2. Let $I \subset A$ be a graded ideal. Then, for all $k$ and for all $j \leq k$, we have

$$
\beta_{i, i+j}(I)=\beta_{i, i+j}\left(I_{\leq k}\right)
$$

Proof. There is an isomorphism of graded $K$-vector spaces $\operatorname{Tor}_{i}^{R}(K, I)$ $\cong H_{i}(\mathbf{x} ; I)$, where $H_{i}(\mathbf{x} ; I)$ denotes the Koszul homology of $I$ with respect to the sequence $\mathbf{x}=x_{1}, x_{2}, \ldots, x_{n}$ of the variables. Thus, we have

$$
\beta_{i, i+j}(I)=\operatorname{dim}_{K} H_{i}(\mathbf{x} ; I)_{i+j} .
$$

A homogeneous cycle $c$ of degree $i+j$ representing a homology class in $H_{i}(\mathbf{x} ; I)_{i+j}$ is a linear combination $\sum_{\sigma} a_{\sigma} e_{\sigma}$ of the canonical basis elements $e_{\sigma}=e_{k_{1}} \wedge \ldots \wedge e_{k_{i}}$ with coefficients $a_{\sigma} \in I_{j}$. Thus, we see that $c$ also represents a cycle in $H_{i}\left(\mathbf{x} ; I_{\leq k}\right)_{i+j}$, provided $j \leq k$. In the same way we see that the $i$-boundaries of the Koszul complex for $I$ and $I_{\leq k}$ coincide whenever $j \leq k$. Hence, we have

$$
H_{i}(\mathbf{x} ; I)_{i+j} \cong H_{i}\left(\mathbf{x} ; I_{\leq k}\right)_{i+j}
$$

for $j \leq k$. This proves the assertion as required.

We now prove that graded Betti numbers of a componentwise linear ideal can be determined by the graded Betti numbers of its components.

Proposition 1.3. Suppose that a graded ideal $I \subset A$ is componentwise linear. Then

$$
\beta_{i, i+j}(I)=\beta_{i}\left(I_{\langle j\rangle}\right)-\beta_{i}\left(\mathfrak{m} I_{\langle j-1\rangle}\right)
$$

for all $j$.

Proof. We proceed by induction on the highest degree $t$ of a generator in a minimal set of generators of $I$. If $t=1$, then $I$ is generated by linear forms, and hence has a linear resolution. Thus, in this case the assertion is obvious.

Suppose that $t>1$, and consider the short exact sequence

$$
0 \longrightarrow I_{\leq t-1} \longrightarrow I \longrightarrow I_{\langle t\rangle} / \mathfrak{m} I_{\langle t-1\rangle} \longrightarrow 0
$$

which for each $j$ yields the long exact sequence

(1) $\operatorname{Tor}_{i}\left(K, I_{\leq t-1}\right)_{i+j} \longrightarrow \operatorname{Tor}_{i}(K, I)_{i+j} \longrightarrow \operatorname{Tor}_{i}\left(K, I_{\langle t\rangle} / \mathfrak{m} I_{\langle t-1\rangle}\right)_{i+j}$. 
Since $I_{\leq t-1}$ is generated in degree $\leq t-1$ we may apply our induction hypothesis, and conclude in particular that $\beta_{i, i+j}\left(I_{\leq t-1}\right)=0$ for $j \geq t$. Hence, the sequence (1) entails

$$
\operatorname{Tor}_{i}(K, I)_{i+j}=\operatorname{Tor}_{i}\left(K, I_{\langle t\rangle} / \mathfrak{m} I_{\langle t-1\rangle}\right)_{i+j} \quad \text { for } \quad j \geq t .
$$

Now, we want to check our formula for $\beta_{i, i+j}(I)$. By Lemma 1.2, we have $\beta_{i, i+j}(I)=\beta_{i, i+j}\left(I_{\leq t-1}\right)$ for $j \leq t-1$. Therefore, by induction hypothesis, our formula is true for $j \leq t-1$. In order to prove it for $j \geq t$, we consider the exact sequence

$$
0 \longrightarrow \mathfrak{m} I_{\langle t-1\rangle} \longrightarrow I_{\langle t\rangle} \longrightarrow I_{\langle t\rangle} / \mathfrak{m} I_{\langle t-1\rangle} \longrightarrow 0
$$

which for each $j$ yields the long exact sequence

$$
\begin{array}{r}
\operatorname{Tor}_{i+1}\left(K, I_{\langle t\rangle} / \mathfrak{m} I_{\langle t-1\rangle}\right)_{i+j} \longrightarrow \operatorname{Tor}_{i}\left(K, \mathfrak{m} I_{\langle t-1\rangle}\right)_{i+j} \longrightarrow \operatorname{Tor}_{i}\left(K, I_{\langle t\rangle}\right)_{i+j} \\
\longrightarrow \operatorname{Tor}_{i}\left(K, I_{\langle t\rangle} / \mathfrak{m} I_{\langle t-1\rangle}\right)_{i+j} \longrightarrow \operatorname{Tor}_{i-1}\left(K, I_{\langle t\rangle}\right)_{i+j} .
\end{array}
$$

The Tor-group at the right end of this sequence vanishes for $j \geq t$ since the corresponding module has a $t$-linear resolution. Also the module on the left end of this sequence vanishes for $j=t$. This proves the desired formula for $j=t$, and also shows that the sequence

$$
\operatorname{Tor}_{i}\left(K, I_{\langle t\rangle}\right)_{i+j} \longrightarrow \operatorname{Tor}_{i}\left(K, I_{\langle t\rangle} / \mathfrak{m} I_{\langle t-1\rangle}\right)_{i+j} \longrightarrow 0
$$

is exact for $j \geq t$. However, $\operatorname{Tor}_{i}\left(K, \mathfrak{m} I_{\langle t-1\rangle}\right)_{i+j}=0$ for $j>t$. Here we used the well-known and easy to prove fact that, if an ideal $I$ has a linear resolution, then $\mathfrak{m} I$ has again a linear resolution. Therefore, we have $\operatorname{Tor}_{i}\left(K, I_{\langle t\rangle} / \mathfrak{m} I_{\langle t-1\rangle}\right)_{i+j}=0$ for $j>t$. In view of (2) this implies $\beta_{i, i+j}(I)=0$ for $j>t$. This concludes our proof since $\mathfrak{m} I_{\langle j-1\rangle}=I_{\langle j\rangle}$ for $j>t$.

Corollary 1.4. Let $I \subset A$ be a graded ideal. Then, the following conditions are equivalent:

(i) I is a Gotzmann ideal;

(ii) $\beta_{i, i+j}(I)=\beta_{i, i+j}\left(I^{\text {lex }}\right)$ for all $i$ and $j$;

(iii) $\beta_{i}(I)=\beta_{i}\left(I^{\text {lex }}\right)$ for all $i$. 
Proof. The equivalence of (ii) and (iii) is obvious since by Bigatti [5], Hulett [17] and Pardue [18] we always have $\beta_{i, i+j}(I) \leq \beta_{i, i+j}\left(I^{\text {lex }}\right)$ for any graded ideal $I$ of $A$.

As we already remarked in Example 1.1, any Gotzmann ideal which is generated in one degree has the same graded Betti numbers as the corresponding lexsegment ideal. Thus, the implication (i) $\Rightarrow$ (ii) follows from Proposition 1.3 if we observe that not only all $I_{\langle j\rangle}$, but also all $\mathfrak{m} I_{\langle j\rangle}$ are Gotzmann ideals, as follows from the persistence theorem.

Now assume (ii) is satisfied. In order to prove (i) we show that each $I_{\langle j\rangle}$ is a Gotzmann ideal. It is clear that $\operatorname{dim}\left(I_{\langle j\rangle}\right)_{j}=\operatorname{dim}\left(I_{\langle j\rangle}^{l e x}\right)_{j}$. On the other hand, by assumption we have

$$
\operatorname{dim} I_{j+1} /\left(I_{\langle j\rangle}\right)_{j+1}=\beta_{0, j+1}(I)=\beta_{0, j+1}\left(I^{l e x}\right)=\operatorname{dim} I_{j+1}^{l e x} /\left(I_{\langle j\rangle}^{l e x}\right)_{j+1} .
$$

This implies that $\operatorname{dim}\left(I_{\langle j\rangle}\right)_{j+1}=\operatorname{dim}\left(I_{\langle j\rangle}^{l e x}\right)_{j+1}$. Therefore, by the persistence theorem, $I_{\langle j\rangle}$ is a Gotzmann ideal.

Let $I \subset A$ be an ideal generated by squarefree monomials. Then, for each degree $j$, we write $I_{[j]}$ for the ideal generated by the squarefree monomials of degree $j$ belonging to $I$. We say that $I$ is squarefree componentwise linear if $I_{[j]}$ has a linear resolution for all $j$.

For example, a weakly stable ideal discussed in [4] is squarefree componentwise linear.

The following result says that a squarefree monomial ideal $I \subset A$ is componentwise linear if and only if $I$ is squarefree componentwise linear.

Proposition 1.5. Suppose that $I \subset A$ is an ideal generated by squarefree monomials. Then $I$ is componentwise linear if and only if $I$ is squarefree componentwise linear.

Proof. ("only if") Suppose that $I$ is componentwise linear. Fix $j>0$; then by assumption, $I_{\langle j\rangle}$ has a linear resolution.

The exact sequence

$$
0 \longrightarrow I_{[j]} \longrightarrow I_{\langle j\rangle} \longrightarrow I_{\langle j\rangle} / I_{[j]} \longrightarrow 0
$$

gives rise to the long exact sequence

$$
\longrightarrow \operatorname{Tor}_{i+1}\left(K, I_{\langle j\rangle} / I_{[j]}\right) \stackrel{\alpha_{i}}{\longrightarrow} \operatorname{Tor}_{i}\left(K, I_{[j]}\right) \longrightarrow \operatorname{Tor}_{i}\left(K, I_{\langle j\rangle}\right) \longrightarrow .
$$


Since the ideals considered are generated by monomials, it follows that all the Tor-groups in the exact sequence are multigraded $K$-vector spaces. It follows from Hochster's formulas ([16, Theorem 5.1]) that $\operatorname{Tor}_{i}\left(K, I_{[j]}\right)$ has only squarefree components, that is, $\operatorname{Tor}_{i}\left(K, I_{[j]}\right)_{a}=0$ if one entry of the vector $a$ is $>1$. On the other hand, since all generators of $I_{\langle j\rangle} / I_{[j]}$ have non-squarefree degrees, it follows that $\operatorname{Tor}_{i+1}\left(K, I_{\langle j\rangle} / I_{[j]}\right)$ has only nonsquarefree components. Therefore, since $\alpha_{i}$ is multihomogeneous, $\alpha_{i}$ must be the zero map. But then for each $i$ the map $\operatorname{Tor}_{i}\left(K, I_{[j]}\right) \rightarrow \operatorname{Tor}_{i}\left(K, I_{\langle j\rangle}\right)$ is injective. Since $I_{\langle j\rangle}$ has a linear resolution, the graded $K$-vector space $\operatorname{Tor}_{i}\left(K, I_{\langle j\rangle}\right)$ is concentrated in degree $i+j$. It follows that $\operatorname{Tor}_{i}\left(K, I_{[j]}\right)$ is concentrated in degree $i+j$. Hence $I_{[j]}$ has a linear resolution.

("if") Suppose that $I_{[j]}$ has a linear resolution for all $j$. We will show by induction on $j$ that $I_{\langle j\rangle}$ has a linear resolution for all $j$.

For the lowest degree $t$ for which $I_{t} \neq 0$, there is nothing to show since $I_{\langle t\rangle}=I_{[t]}$. We now assume that the assertion is true for some $j \geq t$. Then $I_{\langle j\rangle}$ has a linear resolution. This implies that $\mathfrak{m} I_{\langle j\rangle}$ has a linear resolution, too. Therefore, we know from the "only if" part that the squarefree part of $\mathfrak{m} I_{\langle j\rangle}$, we call it $L$, has a linear resolution. It is clear that $L$ is also contained in $I_{[j+1]}$. Hence, we get the exact sequence

$$
0 \longrightarrow L \longrightarrow \mathfrak{m} I_{\langle j\rangle} \oplus I_{[j+1]} \longrightarrow I_{\langle j+1\rangle} \longrightarrow 0
$$

where $u \in L$ is mapped to $(u,-u) \in \mathfrak{m} I_{\langle j\rangle} \oplus I_{[j+1]}$.

We noted already that $L$ and $\mathfrak{m} I_{\langle j\rangle}$ have linear resolutions. Furthermore, $I_{[j+1]}$ has a linear resolution by assumption.

From the long exact Tor-sequence which is derived from (3) we deduce that $I_{\langle j+1\rangle}$ has a linear resolution once it is shown that

$$
\operatorname{Tor}_{i}(K, L) \longrightarrow \operatorname{Tor}_{i}\left(K, \mathfrak{m} I_{\langle j\rangle}\right) \oplus \operatorname{Tor}_{i}\left(K, I_{[j+1]}\right)
$$

is injective for all $i$. But this is clear since already the first component of this map is injective because $L$ is the squarefree part of $\mathfrak{m} I_{\langle j\rangle}$; cf. the proof "only if".

Corollary 1.6. Let $I \subset A$ be an ideal which is generated by squarefree monomials and suppose that I is componentwise linear. Then, for each $j,(\mathfrak{m} I)_{[j]}$ has a linear resolution and

$$
\beta_{i, i+j}(I)=\beta_{i}\left(I_{[j]}\right)-\beta_{i}\left((\mathfrak{m} I)_{[j]}\right)
$$


Proof. Since $I$ is componentwise linear, the ideal $(\mathfrak{m} I)_{\langle j\rangle}=\mathfrak{m} I_{\langle j-1\rangle}$ has a linear resolution. Moreover, since $(\mathfrak{m} I)_{[j]}$ is the squarefree part of $(\mathfrak{m} I)_{\langle j\rangle}$, the ideal $(\mathfrak{m} I)_{[j]}$ also has a linear resolution. Now, it follows from the proof of Proposition 1.3 that

$$
\beta_{i}\left(I_{\langle j\rangle}\right)-\beta_{i}\left(\mathfrak{m} I_{\langle j-1\rangle}\right)=\beta_{i}\left(I_{\langle j\rangle} / \mathfrak{m} I_{\langle j-1\rangle}\right)
$$

By the similar technique, we easily obtain the equality

$$
\beta_{i}\left(I_{[j]}\right)-\beta_{i}\left((\mathfrak{m} I)_{[j]}\right)=\beta_{i}\left(I_{[j]} /(\mathfrak{m} I)_{[j]}\right)
$$

for every $j$. Since $I$ is generated by squarefree monomials, we have

$$
I_{\langle j\rangle} / \mathfrak{m} I_{\langle j-1\rangle} \cong I_{[j]} /(\mathfrak{m} I)_{[j]}
$$

Hence, the desired formula follows immediately from Proposition 1.3.

\section{$\S 2$. Combinatorics on componentwise linear ideals}

We now discuss combinatorial application of Corollary 1.6 and, based on [9], present a formula to compute graded Betti numbers of componentwise linear ideals generated by squarefree monomials.

Let $\Delta$ be a simplicial complex on the vertex set $[n]=\{1,2, \ldots, n\}$, i.e., $\Delta$ is a collection of subsets of $[n]$ such that (i) $\{i\} \in \Delta$ for each $1 \leq i \leq n$ and (ii) $F \in \Delta, G \subset F \Rightarrow G \in \Delta$. A subset $F \subset[n]$ is called a $q$-face of $\Delta$ if $F \in \Delta$ with $\sharp(F)=q+1$. Here, $\sharp(F)$ is the cardinality of a finite set $F \subset[n]$. A maximal face of $\Delta$ is also called a facet of $\Delta$. The dimension of $\Delta$ is $\operatorname{dim} \Delta:=\max \{\sharp(F) ; F \in \Delta\}-1$. Let $f_{q}$ be the number of $q$-faces of $\Delta$ and call $f(\Delta)=\left(f_{0}, f_{1}, f_{2}, \ldots\right)$ the $f$-vector of $\Delta$. Let $h(\Delta)=\left(h_{0}, h_{1}, h_{2}, \ldots\right)$ denote the $h$-vector of $\Delta$ defined in, e.g., [6], [15] and [19]. We say that a simplicial complex $\Delta$ is pure if all facets of $\Delta$ have the same cardinality.

Let $\Delta^{*}$ denote the dual simplicial complex of $\Delta$, that is to say,

$$
\Delta^{*}=\{[n]-F ; F \notin \Delta\}
$$

and, for each $q$, write $\Delta^{*}(q)$ for the (pure) subcomplex of $\Delta^{*}$ whose facets are the $q$-faces of $\Delta^{*}$. Moreover, we define $\Delta^{*}(q)^{\prime}$ to be the subcomplex of $\Delta^{*}(q)$ whose facets are those $q$-faces $F$ of $\Delta^{*}$ such that $F$ is not a facet of $\Delta^{*}$. In other words, $\Delta^{*}(q)^{\prime}$ is the $q$-skeleton of $\Delta^{*}(q+1)$. In particular, 
if $\Delta^{*} \neq\{\emptyset\}$ then $\Delta^{*}(-1)=\Delta^{*}(-1)^{\prime}=\{\emptyset\}$. Moreover, if $\Delta^{*}=\{\emptyset\}$ then $\Delta^{*}(-1)=\{\emptyset\}$ and $\Delta^{*}(-1)^{\prime}=\emptyset$.

Let $A=K\left[x_{1}, x_{2}, \ldots, x_{n}\right]$ denote the polynomial ring in $n$ variables over a field $K$ with each $\operatorname{deg} x_{i}=1$ and $I_{\Delta}$ the ideal of $A$ generated by all squarefree monomials $x_{i_{1}} x_{i_{2}} \cdots x_{i_{r}}, 1 \leq i_{1}<i_{2}<\cdots<i_{r} \leq n$, with $\left\{i_{1}, i_{2}, \ldots, i_{r}\right\} \notin \Delta$. We say that the quotient algebra $K[\Delta]:=A / I_{\Delta}$ is the Stanley-Reisner ring of $\Delta$ over $K$. A simplicial complex $\Delta$ is called CohenMacaulay over $K$ if the Stanley-Reisner ring $K[\Delta]$ is Cohen-Macaulay. We refer the reader to [6], [15], [16] and [19] for the detailed information about Cohen-Macaulay complexes.

Suppose now that $\Delta^{*}(q)$ is Cohen-Macaulay for every $q$. Let $d=$ $\operatorname{dim} \Delta^{*}$. Thus $\Delta^{*}(d)^{\prime}=\emptyset$. If $q<d$, then $\Delta^{*}(q)^{\prime}$ is Cohen-Macaulay (in fact, level [14]). Since $\Delta^{*}(q)^{\prime}$ is a Cohen-Macaulay subcomplex of the Cohen-Macaulay complex $\Delta^{*}(q)$ with $\operatorname{dim} \Delta^{*}(q)^{\prime}=\operatorname{dim} \Delta^{*}(q)$, it follows from, e.g., [20] that $h\left(\Delta^{*}(q)^{\prime}\right) \leq h\left(\Delta^{*}(q)\right)$, i.e., $h_{i}\left(\Delta^{*}(q)^{\prime}\right) \leq h_{i}\left(\Delta^{*}(q)\right)$ for each $i$. (Here, $h_{i}(\Delta)$ is the $i$-th component of $h(\Delta)$.) Let $h\left(\Delta^{*}(d)\right)=$ $\left(h_{0}(d), h_{1}(d), h_{2}(d), \ldots\right)$ and, for each $q<d$,

$$
h\left(\Delta^{*}(q)\right)-h\left(\Delta^{*}(q)^{\prime}\right)=\left(h_{0}(q), h_{1}(q), h_{2}(q), \ldots\right) .
$$

Thus, in particular, $h_{0}(d)=1$ and $h_{0}(q)=0$ for each $q<d$.

We are now in the position to state the main results of the present paper.

TheOREm 2.1. (a) The ideal $I_{\Delta} \subset A$ associated with a simplicial complex $\Delta$ is componentwise linear if and only if $\Delta^{*}(q)$ is Cohen-Macaulay for every $q$.

(b) Suppose that $I_{\Delta}$ is componentwise linear. Then

$$
\sum_{i \geq 0} \beta_{i, i+j}\left(I_{\Delta}\right) \lambda^{i}=\sum_{i \geq 0} h_{i}(n-j-1)(\lambda+1)^{i}
$$

for every $j \geq 1$.

Proof. (a) Let $I=I_{\Delta}$. Then, by Proposition 1.5, $I$ is componentwise linear if and only if $I$ is squarefree componentwise linear. Let $\Delta_{j}$ denote the simplicial complex on $[n]$ with $I_{[j]}=I_{\Delta_{j}}$. If $F \subset[n]$, then $F \notin \Delta_{j}$ if and only if there exists a subset $G \subset[n]$ such that $G \subset F, \sharp(G)=j$ and $G \notin \Delta$. In other words, $[n]-F \in\left(\Delta_{j}\right)^{*}$ if and only if there exists a subset $G \subset[n]$ such that $[n]-F \subset[n]-G, \sharp([n]-G)=n-j$ and $[n]-G \in \Delta^{*}$. 
Hence, $\left(\Delta_{j}\right)^{*}=\Delta^{*}(n-j-1)$. Thus, by [9, Theorem 3], $I_{[j]}$ has a linear resolution if and only if $\Delta^{*}(n-j-1)$ is Cohen-Macaulay. Hence, $I_{\Delta}$ is componentwise linear if and only if $\Delta^{*}(q)$ is Cohen-Macaulay for every $q$.

(b) Suppose that $I=I_{\Delta}$ is componentwise linear. Choose the simplicial complexes $\Delta_{j}$ and $\Delta_{j}^{\prime}$ with $I_{[j]}=I_{\Delta_{j}}$ and $(\mathfrak{m} I)_{[j]}=I_{\Delta_{j}^{\prime}}$. We know $\left(\Delta_{j}\right)^{*}=$ $\Delta^{*}(n-j-1)$. Moreover, if $F \subset[n]$, then $F \notin \Delta_{j}^{\prime}$ if and only if there exists a subset $G \subset[n]$ with $G \subset F$ and $\sharp(G)=j$ such that $G-\{k\} \notin \Delta$ for some $k \in G$. In other words, $[n]-F \in\left(\Delta_{j}^{\prime}\right)^{*}$ if and only if there exists a subset $G \subset[n]$ with $[n]-F \subset[n]-G$ and $\sharp([n]-G)=n-j$ such that $([n]-G) \bigcup\{k\} \in \Delta^{*}$ for some $k \notin[n]-G$. Hence, $\left(\Delta_{j}^{\prime}\right)^{*}=\Delta^{*}(n-j-1)^{\prime}$.

Now, $[9$, Theorem 4] together with Corollary 1.6 guarantees that

$$
\begin{aligned}
\sum_{i \geq 0} \beta_{i, i+j}(I) \lambda^{i}= & \sum_{i \geq 0} \beta_{i}\left(I_{[j]}\right) \lambda^{i}-\sum_{i \geq 0} \beta_{i}\left((\mathfrak{m} I)_{[j]}\right) \lambda^{i} \\
= & \sum_{i \geq 0} h_{i}\left(\Delta^{*}(n-j-1)\right)(\lambda+1)^{i} \\
& \quad-\sum_{i \geq 0} h_{i}\left(\Delta^{*}(n-j-1)^{\prime}\right)(\lambda+1)^{i} \\
& =\sum_{i \geq 0} h_{i}(n-j-1)(\lambda+1)^{i}
\end{aligned}
$$

as desired.

We should remark that if $I=I_{\Delta}$ is weakly stable then $\Delta^{*}(q)$ is shellable (e.g., [6, p. 206]) for every $q$. Note that, by Duval [24], $\Delta^{*}(q)$ is CohenMacaulay for every $q$ if and only if $\Delta^{*}$ is sequentially Cohen-Macaulay ([19]).

EXAMPLE 2.2. Let $n=6$ and $I=I_{\Delta}$ the weakly stable ideal generated by $x_{1} x_{2} x_{4}, x_{1} x_{3} x_{4}, x_{1} x_{2} x_{3} x_{5}, x_{2} x_{3} x_{4} x_{5}$, and $x_{2} x_{3} x_{4} x_{6}$. Then $d=2$ and the facets of $\Delta^{*}$ are $\{1,5\},\{1,6\},\{4,6\},\{2,5,6\}$ and $\{3,5,6\}$. The $f$-vector of $\Delta^{*}(2)$ is $f\left(\Delta^{*}(2)\right)=(4,5,2)$ and $h\left(\Delta^{*}(2)\right)=(1,1,0,0)$. Moreover, since $f\left(\Delta^{*}(1)\right)=(6,8), f\left(\Delta^{*}(1)^{\prime}\right)=(4,5), h\left(\Delta^{*}(1)\right)=(1,4,3)$ and $h\left(\Delta^{*}(1)^{\prime}\right)=$ $(1,2,2)$, we have $h\left(\Delta^{*}(1)\right)-h\left(\Delta^{*}(1)^{\prime}\right)=(0,2,1)$. Hence, if $j=3$ then $\beta_{03}+\beta_{14} \lambda+\beta_{25} \lambda^{2}+\cdots=1+(1+\lambda)=2+\lambda$, and if $j=4$ then $\beta_{04}+$ $\beta_{15} \lambda+\beta_{26} \lambda^{2}+\cdots=2(1+\lambda)+(1+\lambda)^{2}=3+4 \lambda+\lambda^{2}$. 
We conclude this paper with a somewhat surprising combinatorial application of [9, Theorem 3]. If $a$ and $i$ are positive integers and if

$$
a=\left(\begin{array}{c}
a_{i} \\
i
\end{array}\right)+\left(\begin{array}{c}
a_{i-1} \\
i-1
\end{array}\right)+\cdots+\left(\begin{array}{c}
a_{j} \\
j
\end{array}\right)
$$

is the $i$-th Macaulay expansion (e.g., [15, p. 15]) of $a$, then we define

$$
\partial_{i-1}(a):=\left(\begin{array}{c}
a_{i} \\
i-1
\end{array}\right)+\left(\begin{array}{c}
a_{i-1} \\
i-2
\end{array}\right)+\cdots+\left(\begin{array}{c}
a_{j} \\
j-1
\end{array}\right) .
$$

Recall that the Kruskal-Katona theorem says that, given a finite sequence $f=\left(f_{0}, f_{1}, \cdots, f_{d-1}\right)$ of positive integers, there exists a simplicial complex $\Delta$ of dimension $d-1$ with $f(\Delta)=f$ if and only if $\partial_{i}\left(f_{i}\right) \leq f_{i-1}$ for every $1 \leq i \leq d-1$.

THEOREM 2.3. Let $\Delta$ be a pure simplicial complex of dimension $d-1$ with $f$-vector $\left(f_{0}, f_{1}, \cdots, f_{d-1}\right)$ and suppose that $\partial_{d-1}\left(f_{d-1}\right)=f_{d-2}$. Then $\Delta$ is Cohen-Macaulay over an arbitrary field.

Proof. Let $E$ denote the exterior algebra of the vector space over $K$ with basis $e_{1}, e_{2}, \ldots, e_{n}$. If $F=\left\{i_{1}, i_{2}, \ldots, i_{q}\right\}$ is a subset of $[n]$ with $1 \leq$ $i_{1}<i_{2} \cdots<i_{q} \leq n$, then $e_{F}:=e_{i_{1}} \wedge e_{i_{2}} \wedge \ldots \wedge e_{i_{q}}$ is called a monomial of $E$ of degree $q$. We may associate each monomial $e_{F}$ of $E$ with the squarefree monomial $\prod_{i \in F} x_{i}$ of $A$. Given a simplicial complex $\Delta$ on $[n]$, we write $J_{\Delta}$ for the ideal of $E$ generated by all monomials $e_{F}$ with $F \notin \Delta$.

Now, let $\Delta$ be a pure simplicial complex on $[n]$ of dimension $d-1$ with $f$-vector $\left(f_{0}, f_{1}, \cdots, f_{d-1}\right)$ and suppose that $\partial_{d-1}\left(f_{d-1}\right)=f_{d-2}$. Let $\Delta^{*}$ be the dual complex of $\Delta$. Since $\Delta$ is pure of dimension $d-1$, the ideal $J_{\Delta^{*}} \subset E$ is generated by monomials of degree $n-d$. By virtue of the squarefree Gotzmann theorem [3, Theorem 4.6] and "higher" KruskalKatona theorem [3, Theorem 4.4], the similar technique as in Example 1.1 (b) enables us to show that the ideal $J_{\Delta^{*}} \subset E$ has a linear resolution. By [16, Theorem 5.1] and [3, Theorem 6.4], it follows that $J_{\Delta^{*}} \subset E$ has a linear resolution if and only if $I_{\Delta^{*}} \subset A$ has a linear resolution. Hence, again by $\left[9\right.$, Theorem 3], $\left(\Delta^{*}\right)^{*}(=\Delta)$ is Cohen-Macaulay as required.

It might be of interest to give a combinatorial proof of the above Theorem 2.3. 


\section{REFERENCES}

[1] A. Aramova and J. Herzog, Koszul cycles and Eliahou-Kervaire type resolutions, J. Algebra, 181 (1996), 347-370.

[2] A. Aramova, J. Herzog and T. Hibi, Squarefree lexsegment ideals, Math. Z., 228 (1998), 353-378.

[3] A. Aramova, J. Herzog and T. Hibi, Gotzmann theorems for exterior algebras and combinatorics, J. Algebra, 191 (1997), 174-211.

[4] A. Aramova, J. Herzog and T. Hibi, Weakly stable ideals, Osaka J. Math., 34 (1997), $745-755$.

[5] A. M. Bigatti, Upper bounds for the Betti numbers of a given Hilbert function, Comm. Algebra, 21 (1993), 2317 - 2334.

[6] W. Bruns and J. Herzog, Cohen-Macaulay Rings, Cambridge University Press, Cambridge, New York, Sydney, 1993.

[7] W. Bruns and J. Herzog, Semigroup rings and simplicial complexes, J. Pure and Appl. Algebra, 122 (1997), 185-208.

[8] H. Charalambous and E. G. Evans, Resolutions obtained by iterated mapping cones, J. Algebra, 176 (1995), 750-754.

[9] J. A. Eagon and V. Reiner, Resolutions of Stanley-Reisner rings and Alexander duality, J. Pure and Appl. Algebra, 130 (1998), 265-275.

[10] D. Eisenbud, Commutative Algebra with a View Toward Algebraic Geometry, Springer-Verlag, 1995.

[11] S. Eliahou and M. Kervaire, Minimal resolutions of some monomial ideals, J. Algebra, 129 (1990), 1-25.

[12] G. Gotzmann, Eine Bedingung für die Flachheit und das Hilbertpolynom eines graduierten Ringes, Math. Z, 158 (1978), 61-70.

[13] M. Green, Restrictions of linear series to hyperplanes, and some results of macaulay and Gotzmann, in "Algebraic Curves and Projective Geometry", Springer Lect. Notes in Math., No. 1389, 1988, pp. 76-89.

[14] T. Hibi, Level rings and algebras with straightening laws, J. Algebra, 117 (1988), 343-362.

[15] T. Hibi, Algebraic Combinatorics on Convex Polytopes, Carslaw Publications, Glebe, N.S.W., Australia, 1992.

[16] M. Hochster, Cohen-Macaulay rings, combinatorics, and simplicial complexes, in "Ring Theory II" (B. R. McDonald and R. Morris, Eds.), Lect. Notes in Pure and Appl. Math., No. 26, Dekker, New York, 1977, pp. 171-223.

[17] H. A. Hulett, Maximum Betti Numbers for a given Hilbert function, Comm. Algebra, 21 (1993), 2335-2350.

[18] K. Pardue, Nonstandard Borel-fixed ideals, Ph. D. thesis, Brandeis University, 1994.

[19] R. P. Stanley, Combinatorics and Commutative Algebra, Second Ed., Birkhäuser, Boston, Basel, Stuttgart, 1996.

[20] R. P. Stanley, A monotonicity property of h-vectors and $h^{*}$-vectors, Europ. J. Combin., 14 (1993), 251-258. 
[21] N. Terai and T. Hibi, Computation of Betti numbers of monomial ideals associated with cyclic polytopes, Discrete and Comput. Geom., 15 (1996), 287-295.

[22] N. Terai and T. Hibi, Computation of Betti numbers of monomial ideals associated with stacked polytopes, Manuscripta Math., 92 (1997), 447-453.

[23] N. Terai and T. Hibi, Alexander duality theorem and second Betti numbers of Stanley-Reisner rings, Adv. in Math., 124 (1996), 332-333.

[24] A. M. Duval, Algebraic shifting and sequentially Cohen-Macaulay simplicial complexes, Electronic J. Combinatorics, 3 (1996), \#R 21.

\author{
Jürgen Herzog \\ FB 6 Mathematik und Informatik \\ Universität-GHS-Essen \\ Essen 45117, Germany \\ juergen.herzog@uni-essen.de \\ Takayuki Hibi \\ Department of Mathematics \\ Graduate School of Science \\ Osaka University \\ Toyonaka, Osaka 560-0043, Japan \\ hibi@math.sci.osaka-u.ac.jp
}

